\title{
Cathodoluminescence Measurements at Liquid Helium Temperature of Poly- and Monocrystalline ZnO Films
}

\author{
B.S. Witkowski ${ }^{a}$, Ł. WAChnicki $^{a}$, R. JAkięa ${ }^{a}$, E. Guziewicz $^{a}$ And M. Godlewski ${ }^{a, b}$ \\ ${ }^{a}$ Institute of Physics, Polish Academy of Sciences, al. Lotników 32/46, 02-668 Warsaw, Poland \\ ${ }^{b}$ Dept. of Mathematics and Natural Sciences College of Science, Cardinal S. Wyszyński University \\ Dewajtis 5, 01-815 Warsaw, Poland
}

\begin{abstract}
Scanning electron microscopy, cathodoluminescence and secondary ion mass spectroscopy investigations are used to study an inter-link between structural quality, elements distribution and light emission properties of $\mathrm{ZnO}$ poly- and monocrystalline films grown by the atomic layer deposition. Cathodoluminescence and scanning electron microscopy investigations were performed at liquid helium temperature for four different types of $\mathrm{ZnO}$ films deposited on different substrates.
\end{abstract}

PACS: 78.60.Hk, 77.55.hf, 68.37.Hk, 61.72.Ff

\section{Introduction}

Zinc oxide is a II-VI semiconductor, which has a direct energy gap of about $3.37 \mathrm{eV}$ at room temperature [1] and high exciton binding energy $(60 \mathrm{meV})$. High transparency and high $n$-type conductivity allow application of $\mathrm{ZnO}$ as an electrode in solar cells [2]. $\mathrm{ZnO}$ was also tested for applications as an active part of transparent transistors [3], as selector in cross-bar memories [4], etc. Due to these applications $\mathrm{ZnO}$ is considered now as an attractive material for the construction of photovoltaic, electronic and optoelectronic devices.

In the recent work we showed that monocrystalline $\mathrm{ZnO}$ layers can be deposited by the atomic layer deposition (ALD) at fairly low temperature of $300^{\circ} \mathrm{C}$ and lower [5]. In the present work we discuss results of low temperature cathodoluminescence (CL) measurements of $\mathrm{ZnO}$ films grown on four different types of substrates. CL images are taken from the same regions as scanning electron microscopy (SEM) ones. This enables to get information not only about uniformity of the films studied, but also on an inter-link between microstructure and light emission properties. We can also evaluate role of local potential fluctuations, since these fluctuations result in exciton localization and shifts of recombination energy.

\section{Measurement methods}

In our investigations we used SEM Hitachi SU-70 with the GATAN MONO CL3 system. In the addition to planar scans of the films, cross-sections of the samples are also investigated. CL was detected from line scans, in which we collected emission spectra along the samples cross-sections. All SEM and CL measurements were performed at temperature of $5 \mathrm{~K}$ using $15 \mathrm{kV}$ accelerating voltage. CL detection was set at the edge luminescence of zinc oxide, which dominated the emission spectrum from our ALD-grown films.

$\mathrm{ZnO}$ samples were grown by the ALD at the temperature of $300^{\circ} \mathrm{C}$ using organic zinc precursor (diethyl zinc) and deionized water. Growth parameters were optimized to get monocrystalline films on lattice matched substrates. Further details are given in Ref. [5].

SIMS investigations were performed at room temperature using Caneca XXX system.

\section{Results and discussion}

Four types of $\mathrm{ZnO}$ samples are investigated. These samples were grown at the same conditions of the ALD process on four different substrates - sapphire, GaAs, $\mathrm{Si}$ and nearly lattice matched GaN.

In Fig. 1 we show SEM images taken at cross-sections of the samples studied. In three cases a polycrystalline structure of the $\mathrm{ZnO}$ films is observed. A columnar growth mode of $\mathrm{ZnO}$ is observed. In the case of nearly lattice matched GaN substrate the X-ray diffraction (XRD) investigations (discussed in Ref. [5]) show that columns have identical orientations and that the $\mathrm{ZnO}$ film obtained is monocrystalline.

CL measurements were performed along the lines indicated on each image. Results of these investigations are shown in Fig. 2. Surprisingly the CL intensity is not following the lattice mismatch condition between the substrate and $\mathrm{ZnO}$ films. This is particularly observed for the films grown on sapphire, the substrate successfully used in the GaN technology.

The data are shown in two ways. First (left images) as horizontal lines in which bright pixels mean high CL 


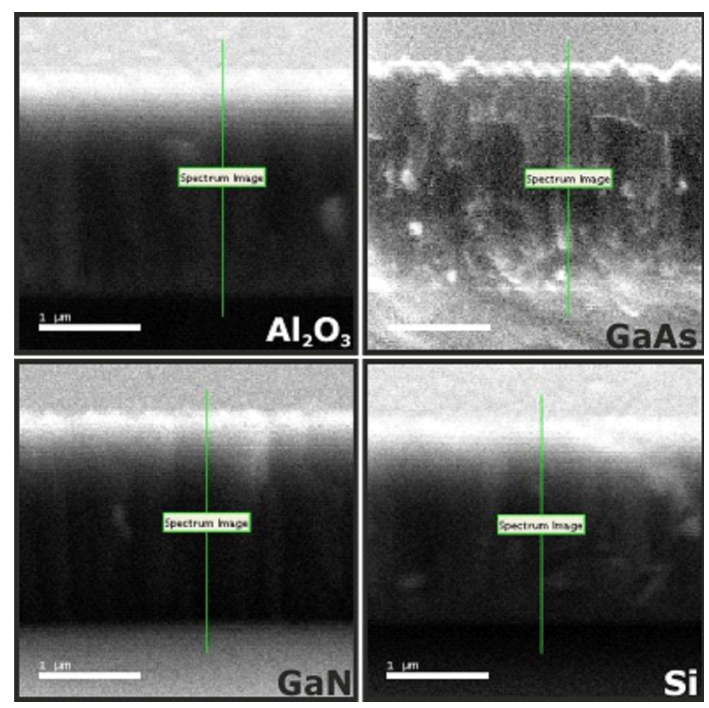

Fig. 1. SEM images of cross-sections of the samples grown on four different substrates. In each image we marked line along which CL spectra were measured.

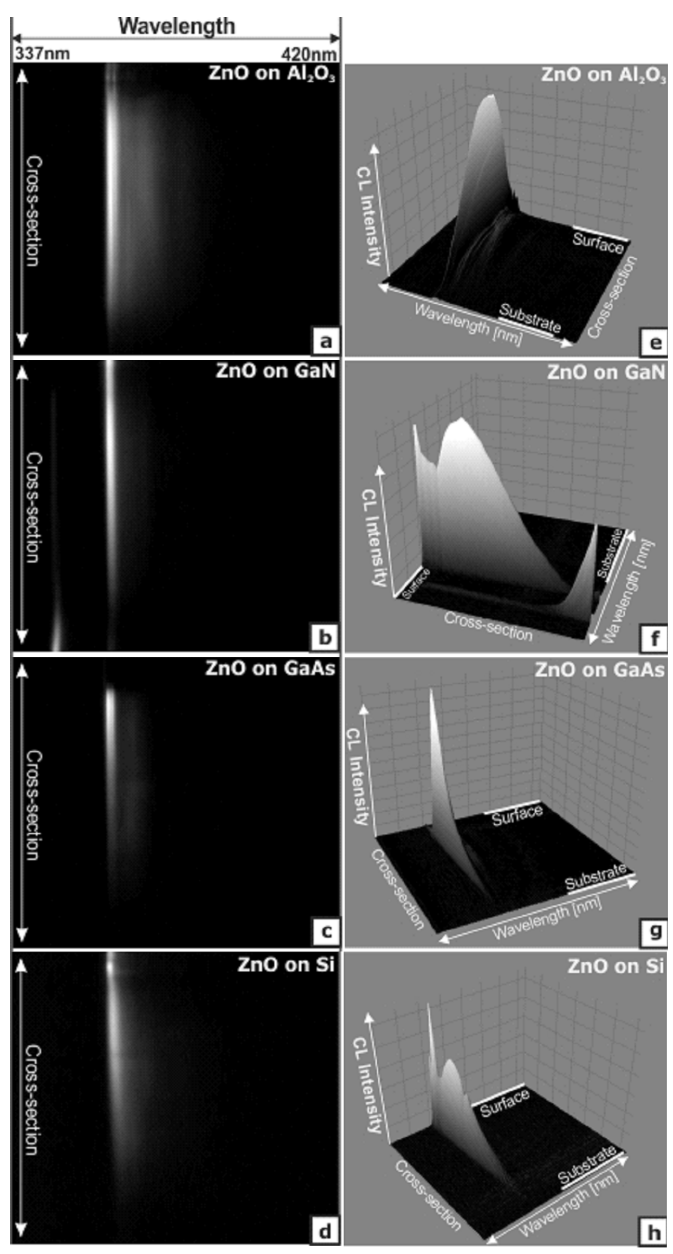

Fig. 2. (a), (b), (c), (d) CL images obtained from the measurements along the cross-sections, (e), (f), (g), (h) the same images as three-dimensional plots. intensity. In the right images $\mathrm{CL}$ is shown as $3 \mathrm{D}$ images - intensity of the band edge emission versus emission wavelength and position on the cross-section.

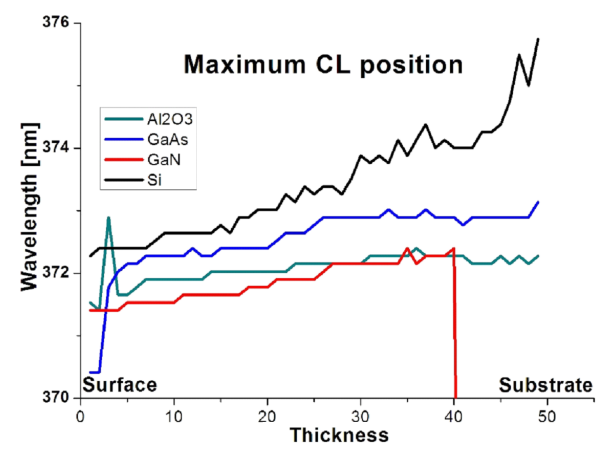

Fig. 3. Dependence of the CL emission wavelength on the position along the cross-sections of the samples. For the latter an arbitrary unit is used since thickness of films slightly varied from sample to sample.

In addition to large variations of the CL intensity the images also show (see Fig. 3) shifts of the wavelength position of the band edge CL maximum along the cross-sections. Such shifts indicate different stress conditions in the samples studied and along their cross-sections.

The largest shift of the CL maximum position for the $\mathrm{ZnO} / \mathrm{Si}$ corresponds to the large lattice mismatch between $\mathrm{ZnO}$ and Si. The smallest shift of the CL position is detected for the samples grown on the nearly lattice matched GaN and surprisingly on the $\mathrm{Al}_{2} \mathrm{O}_{3}$ and $\mathrm{GaAs}$ substrates. The latter observation is fairly surprising. Moreover, Fig. 3 shows that peak position of the band gap emission is different in each of the cases.

The dependence of the CL intensity for each case studied is similar. Band edge emission is weak or is not observed at the interface to the substrate and then rises, reaches the maximum intensity and drops in the intensity or changes character at the surface. The latter (change of the emission energy at the interface) is observed for the samples grown on $\mathrm{Si}, \mathrm{Al}_{2} \mathrm{O}_{3}$ and $\mathrm{GaN}$ substrates, which we first tentatively related to the consequences of donor type impurities floating on the growth front.

We performed secondary ion mass spectroscopy (SIMS) investigations to verify the above given hypothesis. Figure $4 \mathrm{a}$ (data for $\mathrm{ZnO} / \mathrm{Al}_{2} \mathrm{O}_{3}$ ) shows a high content of aluminum and hydrogen near the interface region of the sample. We thus propose that the incorporation of aluminum into the structure results in a faster layer relaxation (CL intensity rises fast once the excitation is moved from the interface region) and also introduces shallow donor states at the surface area, which manifests itself in the appearance of a new emission band, which we can see in Fig. 2a and e.

This extra emission band, likely of the donor bound exciton origin, is observed at a longer wavelength than the $\mathrm{ZnO}$ edge emission. The presence of Al-related emission band in $\mathrm{ZnO}$ was described in [6]. 


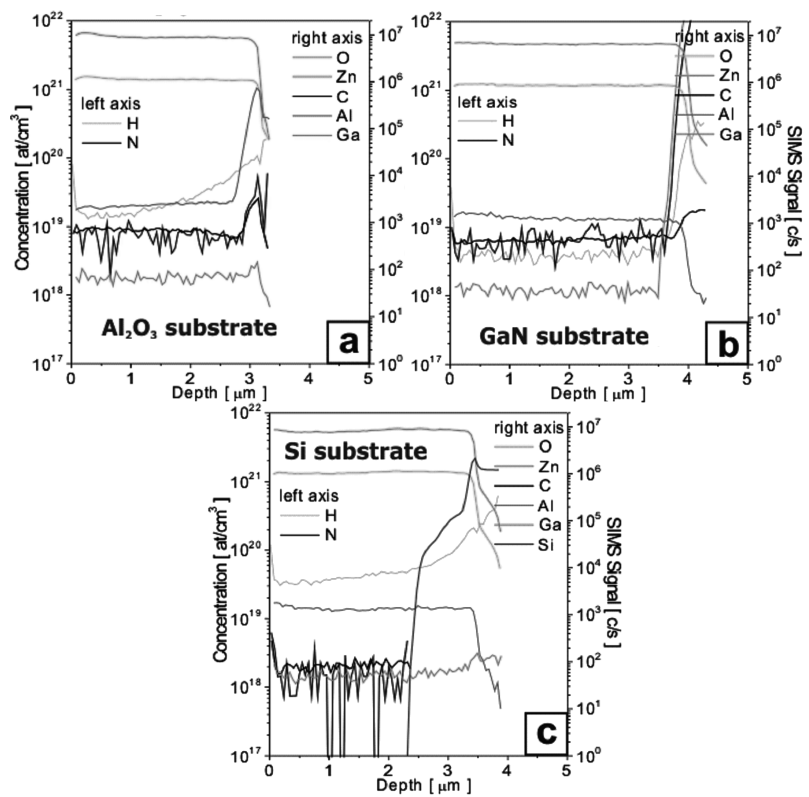

Fig. 4. SIMS results for the $\mathrm{ZnO}$ samples grown by the ALD on four different substrates.

For the samples grown on a Si substrate and GaN template, there is the additional CL peak at the position shifted towards the higher energy detected from the surface close region. SIMS investigations indicate that this extra CL peak may be related to the increased content of hydrogen at the surface. This is only a tentative explanation of the new emission band. Another possibility is modification of surface states, which requires further investigation.

\section{Summary}

CL measurements along the cross-sections provide information about the lattice mismatch and the lattice relaxation along the layer thickness. The present investigations indicate that modifications of stress conditions, dopants distribution, etc., depend on the type of the substrate used in the ALD process. This is rather unexpected result, since it is commonly claimed that the ALD process is not epitaxial and the film grown has properties independent of the substrate used.

\section{Acknowledgments}

The research was partly supported by the European Union within European Regional Development Fund, through the grant of the Innovative Economy (POIG.01.01.02-00-008/08).

\section{References}

[1] C. Klingshirn, Phys. Status Solidi B 244, 3027 (2007).

[2] M. Godlewski, E. Guziewicz, G. Łuka, T. Krajewski, M. Łukasiewicz, Ł. Wachnicki, A. Wachnicka, K. Kopalko, A. Sarem, B. Dalati, Thin Solid Films 518, 1145 (2009).

[3] S. Gieraltowska, L. Wachnicki, B.S. Witkowski, M. Godlewski, E. Guziewicz, Thin Solid Films, to be published.

[4] N. Huby, G. Tallarid, M. Kutrzeba, S. Ferrari, E. Guziewicz, Ł. Wachnicki, M. Godlewski, Microelectron. Eng. 85, 2442 (2008).

[5] Ł. Wachnicki, T. Krajewski, G. Łuka, B. Witkowski, B. Kowalski, K. Kopalko, J.Z. Domagala, M. Guziewicz, M. Godlewski, E. Guziewicz, Thin Solid Films 518, 4556 (2010).

[6] H.P. He, H.P. Tang, Z.Z. Ye, L.P. Zhu, B.H. Zhao, L. Wang, X.H. Li, Appl. Phys. Lett. 90, 023104 (2007). 